\title{
Taurus Tunable Filter — Seven Years of Observing
}

\author{
J. Bland-Hawthorn ${ }^{1}$ and L. Kedziora-Chudczer ${ }^{2}$ \\ ${ }^{1}$ Anglo-Australian Observatory, PO Box 296, Epping 2121, Australia \\ jbh@aao.gov.au \\ 2 Dept. of Astrophysics \& Optics, School of Physics, University of New South Wales, \\ Sydney 2052, Australia \\ lkedzior@physics.usyd.edu.au \\ Received 2002 July 22, accepted 2003 May 6
}

\begin{abstract}
The Taurus Tunable Filter (TTF) has now been in regular use for seven years on the AngloAustralian Telescope (AAT). The instrument was also used for three years (1996-1999) on the William Herschel Telescope (WHT). We present a brief review of the different applications in order to illustrate the versatility of tunable filters in optical/IR spectrophotometric imaging. Tunable filters are now either planned or under development for 6-10 m class telescopes which ensures their use for years to come.
\end{abstract}

Keywords: Galaxies — Galactic Nebulae — Quasars

\section{Introduction}

We provide a short review of the first seven years of observing with the Taurus Tunable Filter at the AAT; for the first three years the TTF was also used at the WHT. The instrument has had most of its use on the AAT where it gets between $10 \%$ and $15 \%$ of the telescope time scheduled by the UK and Australian time assignment committees (PATT and ATAC). In a nutshell, the TTF allows for wide-field $\left(10^{\prime}\right)$ spectrophotometric imaging from $370 \mathrm{~nm}$ to $1000 \mathrm{~nm}$ with resolving powers generally in the range 100-1000. An important feature of the instrument is the use of charge shuffling synchronized to band switching in order to greatly suppress systematic errors associated with conventional imaging. Another aspect of the TTF is time series readout coupled to band switching which has led to important new work on compact variable sources. Technical accounts of the instrument, and its related charge shuffling modes, can be found in Appendices A and B.

There have been several recent reviews on tunable filters including Bland-Hawthorn (2000 a,b), Jones (2001 a) and Jones et al. (2001). Recent scientific reviews can be found in Veilleux et al. (2002) and Veilleux (2002a,b). A list of largely unexplored science areas with tunable filters is given in Bland-Hawthorn et al. (2001).

The purpose of this review is to stress the versatility of tunable filters in observational astronomy. The Taurus focal reducer has also produced important results in its other modes of operation (see Appendix A): polarimetry, Fabry-Perot 'staring' and emission line scanning, and most recently multi-object spectroscopy (e.g. Riddick, Lucas \& Roche 2003). But here we concentrate specifically on the TTF mode of operation.

All observations discussed here were undertaken on $4 \mathrm{~m}$ telescopes, often in non-ideal observing conditions. These authors eagerly anticipate the tunable filters which are planned or under way for the new generation optical/ IR telescopes on superb sites. These include, inter alia, the Osiris tunable filter on the Grantecan $10.2 \mathrm{~m}$ telescope (Cepa et al. 2002), a possible tunable filter within FORS on the VLT, the proposed Maryland Magellan tunable filter (MMTF), and the tunable filter within the Goodman spectrograph on the SOAR $4 \mathrm{~m}$ telescope. When one reviews the results below, it is important to keep in mind that the new generation of tunable filters need only improve on one of the following - site conditions, instrument performance, field of view, pixel sampling, telescope aperture to achieve a major gain.

\section{Surveys of Star Forming Galaxies}

\subsection{Field Galaxies}

Tunable filters are ideally suited to surveys of star forming galaxies in different environments. The object selection is based on the property we are trying to measure, i.e. the star formation rate via the $\mathrm{H} \alpha$ line. This was the principle behind the TTF Field Galaxy Survey which was the basis of D.H. Jones' thesis (2001). He obtained photometric $\mathrm{H} \alpha$ data and restricted $\mathrm{H} \beta$ data on four clusters and nine field positions. All observations were highly successful in identifying line emitting galaxies, typically $10-40$ objects above $3 \sigma$ per pointing (although rather fewer in $\mathrm{H} \beta$ ), finding many more objects than comparable studies with conventional imaging techniques (e.g. Hu \& McMahon 1996; Thommes et al. 1998). The first part of the TTF Field Galaxy Survey was published in Jones \& Bland-Hawthorn (2001).

Recent evidence suggests a decline in the volumeaveraged star formation rate (SFR) with the advance of cosmic time since $z \sim 1$. The survey set out to derive $\mathrm{H} \alpha$ luminosity functions in three discrete wavelength intervals at $z=0.08,0.24$, and 0.39 . One of the interesting surprises was the discovery of a population of compact sources found to have moderate amounts of $\mathrm{H} \alpha$ emission: some of these sources would have been dismissed as stars in earlier photographically selected surveys. 
The TTF Field Galaxy Survey measures volumeaveraged SFR at intermediate redshift that are a factor of 2-3 times higher than those measured from multi-slit or multi-fibre surveys. Since these results have been largely confirmed by the CADIS emission line survey (Hippelein et al. 2003) and the SUBARU emission line survey (Fujita et al.2003), it is likely that the restricted apertures of fibres and slits have underestimated the total $\mathrm{H} \alpha$ line flux at low redshift.

In a new study of galaxies in the Canada-France Redshift Survey (CFRS), Tresse et al. (2002) combine the TTF Field Galaxy Survey measurements with their ISAAC/VLT H $\alpha$ luminosity function at $z \sim 1$ (see Fig. 1). They find that the comoving $\mathrm{H} \alpha$ luminosity density increases by a factor of 12 from $z=0.2$ to $z=1.3$. Their results confirm a strong rise of the SFR at $z<1.3$ proportional to $(1+z)^{4.1 \pm 0.3}$.

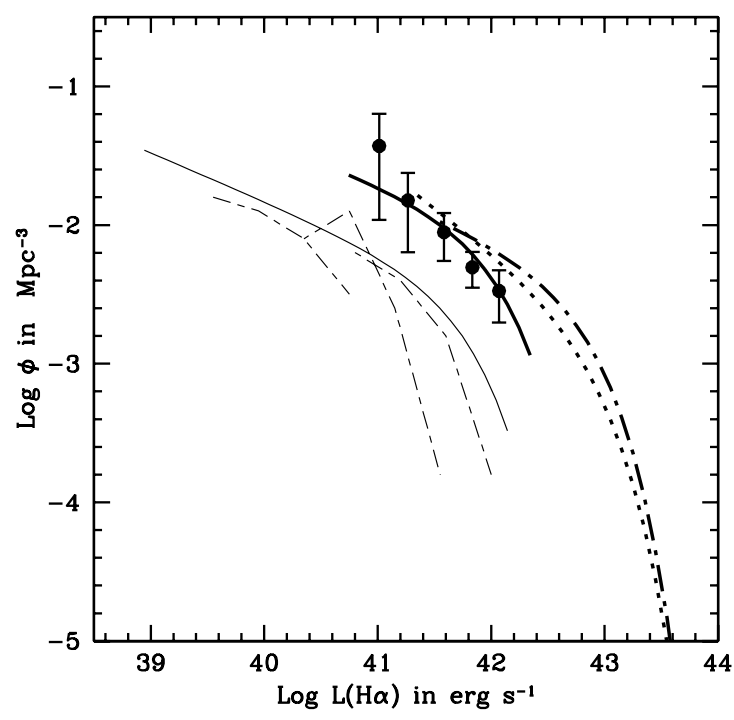

Figure 1 The evolution of the $\mathrm{H} \alpha$ luminosity function with redshift taken from Tresse et al. (2001; Fig. 13). The filled circles are the data points from their ISAAC/VLT survey. The short-long dashed curves are the preliminary $\mathrm{H} \alpha \mathrm{LFs}$ from Jones \& Bland-Hawthorn (2001) from left to right respectively at $z=0.08, z=0.24, z=0.40$. The other curves are defined in Tresse et al. (2001).
Tober et al. (2003; see also Glazebrook 1997) carried out a deep survey of 20 square arcminutes in the Hubble Deep Field North (HDF-N) using the Taurus Tunable Filter and William Herschel Telescope to observe in contiguous sequences of narrow band slices in the 710-910 nm region of the spectrum. These authors sampled almost 70 narrowband images in four redshift intervals. In this way, one gets a multiplicity effect in that the same images can be used to find different sources in several emission lines at different redshifts, thereby allowing for a derivation of the SFR over a wide range in redshift. The data, which were taken during four long dark nights at the WHT, have excellent image quality with $\mathrm{psf} \approx 0.7^{\prime \prime}$ FWHM after combining the full data set.

Cross-matching with Keck spectroscopy of the HDF-N results in a line-luminosity limited sample, with very highly complete redshift identification, containing [OII], $\mathrm{H} \beta$ and $\mathrm{H} \alpha$ emitters over the range $z=0.3-0.9$. From this direct SFR selected sample they reconstruct, via maximum likelihood techniques, the star formation history of the Universe to $z=1$. Their study finds no evidence for any new population of low-luminosity strong-lined objects to an approximate flux limit of $2 \times 10^{-20} \mathrm{~W} \mathrm{~m}^{-2}$ and covering a cosmological volume of $1000 \mathrm{Mpc}^{3}$ at $z=1$. From their complete narrow-band sample of a single sight line, Tober et al. find a star formation history consistent with earlier estimates from broad-band selected samples.

\subsection{Clusters}

Until now, identifying the majority of cluster members from a combination of kinematics and a colour-magnitude diagram has been difficult, particularly for blue objects, due to contamination of sightline galaxies. But TTF observations can be used to identify faint cluster members unambiguously, in particular those with line emission. These objects are typically blue so the TTF is ideally suited to unscrambling the region of contamination (see Fig. 2(Right)). We find that $\mathrm{k}+\mathrm{a}$ galaxies (Dressler \& Gunn 1983) can also be identified from $\mathrm{H} \alpha$ absorption (Jones \& Bland-Hawthorn 1999, Fig. 1).
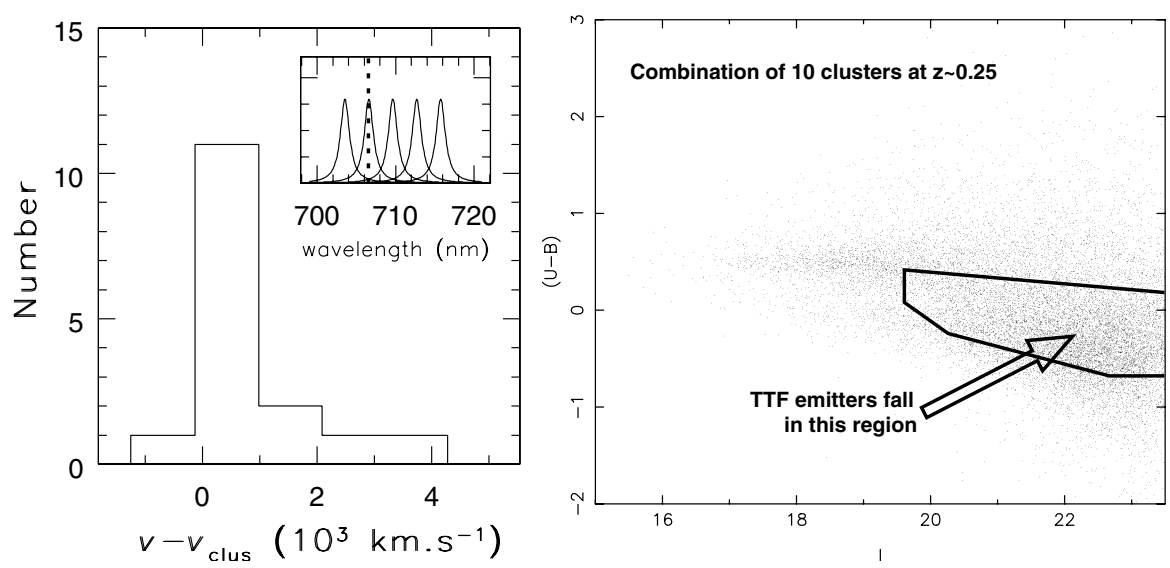

Figure 2 Left - Distribution of emission line objects from a $z=0.4$ cluster field if all of the emission is assumed to be redshifted $\mathrm{H} \beta$. The inset shows the TTF passbands relative to the wavelength of redshifted $\mathrm{H} \beta$ (dotted line). Right - Illustration of where the line emitters fall in a cluster colour-magnitude diagram. The cluster data are from Smail et al. (1998). 


\begin{tabular}{|c|c|c|c|}
\hline $23+2$ & \begin{tabular}{l|}
15510 \\
0.03
\end{tabular} & 150 & $\begin{array}{l}5831 \\
0.16\end{array}$ \\
\hline $31 \angle$ & 0.17 & 152 & $\begin{array}{l}1869 \\
0.06\end{array}$ \\
\hline 45 & $\begin{array}{l}1160 \\
0.53\end{array}$ & 165 & $\begin{array}{l}513 \\
0.03\end{array}$ \\
\hline 58 & $\begin{array}{l}4023 \\
0.61\end{array}$ & 180 & $\begin{array}{l}2544 \\
0.83\end{array}$ \\
\hline 73 & $\begin{array}{l}2656 \\
0.69\end{array}$ & 188 & $\begin{array}{l}1665 \\
0.8\end{array}$ \\
\hline 124 & $\begin{array}{l}4643 \\
0.27\end{array}$ & 210 & $\begin{array}{l}15579 \\
0.43\end{array}$ \\
\hline 128 & $\begin{array}{l}1298 \\
0.36\end{array}$ & 245 & $\begin{array}{l}752 \\
0.47 \\
\end{array}$ \\
\hline 135 & $\begin{array}{l}2055 \\
0.75\end{array}$ & $248 K$ & $\begin{array}{l}2616 \\
0.82\end{array}$ \\
\hline $\begin{array}{ll}1 \\
700 & 710\end{array}$ & 720 & $\begin{array}{rr}700 & 710 \\
\end{array}$ & 720 \\
\hline
\end{tabular}

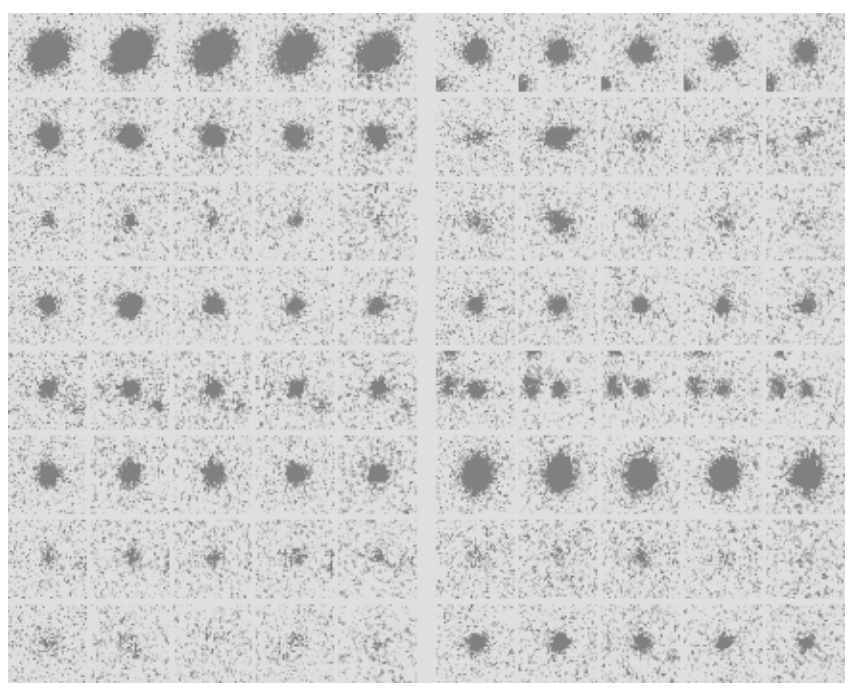

Figure 3 TTF spectra (Left) and scans (Right) for a sample of emission-line cluster candidates. The number on the left of each panel is the object ID, the numbers on the right are the flux in counts (top) and shape classification parameter (bottom; $1=$ stellar, $0=$ galaxian). No attempt has been made to reject possible fore/background contaminants on the basis of appearance or redshift. The vertical dotted line denotes the cluster redshift from Keck spectra of $\sim 10$ galaxies in the field. The wavelength shift of spectra between objects is due to the off-axis phase effect across the TTF field (Bland-Hawthorn \& Jones 1998).

In Figure 2 (Left) we demonstrate the power of TTF to find line emitters even in poor seeing $\left(2^{\prime \prime}\right)$. Dalcanton (1996) and Zaritsky et al. (1997) use CCD drift scanned images to identify clusters through enhanced surface brightness fluctuations out to $z \approx 1.1$. For one cluster at $z=0.45$, in just 2 hours, we identified 10 cluster members at the AAT in $2^{\prime \prime}$ seeing, compared to 6 members in 1.5 nights using the Keck LRIS spectrograph.

For the rich cluster A3665 (AC 106), first studied by Couch \& Newell (1984), Zaritsky \& Jones identify $40 \mathrm{H} \alpha$ emitting candidates above $3 \sigma$, with many more candidates at lower thresholds (down to 0.03 solar masses per year at $z=0.25$ ), in just four hours. Example TTF scans are shown in Figure 3 (Right), with the $\mathrm{H} \alpha$ line profiles in Figure 3 (Left).

To date, average SFR in $z=0.2-0.5$ clusters appear to be quite low compared to the field (cf. Couch et al. 2001).

\subsection{Quasar Environment}

Baker et al. (2001) used the [OII] $372.7 \mathrm{~nm}$ line to detect starforming galaxies in the vicinity of MRC B0450-221. Many emission line galaxies can be visible over the whole field (down to a few solar masses per year). Their projected separations from the quasar range from 200 to $700 \mathrm{kpc}$. A nice demonstration of the power of TTF imaging is the detection of very faint extended [OII] emission along the radio axis of the quasar.

\subsection{Quasar Sight Lines}

Francis et al. (2001 a,b) have continued their study of the 'Francis cluster', an extended ensemble of galaxies at $z=2.38$ that was originally identified from the damped Ly $\alpha$ absorption occurring at the same redshift in a paired quasar sightline. In this study, they look for Ly $\alpha$ emission from star forming galaxies within the ensemble. It is clear that the future holds great promise for using quasars to identify gas-rich starforming environments at high redshift.

\section{Energetic Gas in Clusters}

There have been claims of EUV excesses in clusters which, if true, would suggest that $10^{5}-10^{6} \mathrm{~K}$ gas accounts for a large fraction of missing baryons (Lieu et al. 1999; Bowyer et al. 1999). EUV emission is exceedingly difficult to detect at the Earth: it is too hot to be detected optically, too cool to be seen by X-ray satellites, and undergoes molecular line absorption as it propagates through the interstellar medium (ISM).

Using the EUVE satellite, Lieu et al. (1999) claim a direct EUV detection of the cluster Abell 1795. Maloney \& Bland-Hawthorn (2001) found that such a strong EUV field would ionise the molecular and $\mathrm{HI}$ disks of all spirals in the cluster to such an extent that they should all be $\mathrm{H} \alpha$ bright. TTF observations of the cluster were kindly taken by A. Edge in 'straddle shuffle' (Maloney \& BlandHawthorn 2001) mode in order to subtract the continuum light of the cluster very accurately. Only very faint $\mathrm{H} \alpha$ emission was found in the cluster spirals.

Jaffe \& Bremer (2000) used 'on-off' charge shuffling in order to detect very faint levels of $\mathrm{H} \alpha$ emission in cooling flow clusters. In the case of Abell 2597, they find a nebula extending over $50-60 \mathrm{kpc}$ from the cluster centre. A. Edge and collaborators (in preparation) have looked at a large sample of cooling flow clusters and detect optical line emission in all cases.

\section{Gravitational Lensing}

Tunable filters are ideally suited to observing extended sources, in particular irregularly shaped sources with no 
axis of symmetry. Earlier work with D. H. Jones on star formation in clusters occasionally turned up spectacular gravitational lenses throughout the cluster. As seen through the TTF, these sources can have high contrast because the background object is commonly a highredshift starforming galaxy which emits strongly in emission lines. Hewett et al. (2000) used the TTF to detect extended emission in a galaxy-galaxy gravitational lens due to an intermediate-redshift elliptical galaxy lensing a $z=3.59$ star forming galaxy. They were following up a suggestion by Miralde-Escudé \& Lehar (1992) that the surface density of high-redshift starforming galaxies is so high, galaxy-galaxy lensing should be relatively common.

\section{Galaxies}

The tunable filter has the major advantage of providing detailed spectrophotometric information over a larger field of view (FOV) than that of other 3D instruments. It would be very difficult and very expensive to build an integral field spectrograph which could provide the same field and the same photometric integrity at low light levels. The tunable filter is therefore ideally suited to studying nearby galaxies where the line-emitting gas extends over several arcminutes. This ionised material is an excellent probe of the phenomena taking place in the core of starburst and active galaxies, and can be used to quantify the impact of nuclear and star formation activity on the environment and vice versa. Once again, the reader is encouraged to look at recent reviews by Veilleux (2002 a,b).

\subsection{Radio Galaxies}

Tadhunter and collaborators have undertaken a comprehensive survey of extended ionised haloes around powerful radio galaxies (Tadhunter et al. 2000; SolorzanoInarrea et al. 2002; Solorzano-Inarrea \& Tadhunter 2003). It is well known that optical line emission often aligns with the radio axis, as these authors find in all cases. However more surprising was the frequent occurrence of optical emission perpendicular to the radio axis. In Figure 4, the optical emission in Coma A extends over more than $150 \mathrm{kpc}$ in an egg-shaped nebula (Tadhunter et al. 2000). Remarkably, the faint outer nebula emission matches the extended outer radio lobe over these same scales.

The frequent match-up between optical, radio and $\mathrm{X}$-ray emission in radio galaxies, Seyfert and starburst galaxies is a topic of great interest (see below). In the case of low power radio jets, the emission is thought to arise from synchrotron emission, whereas in high power radio jets, the $\mathrm{X}$-rays appear to arise from inverse Compton emission (Birkinshaw et al. 2001).

\subsection{Quasar Nebulae}

Shopbell et al. (1999) recently published a remarkable extended nebula surrounding the X-ray selected quasar, MR 2251-178. The summed image in Figure 5 is one of the faintest extended emission line images ever published. The nebula, which extends over more than $200 \mathrm{kpc}$, shows

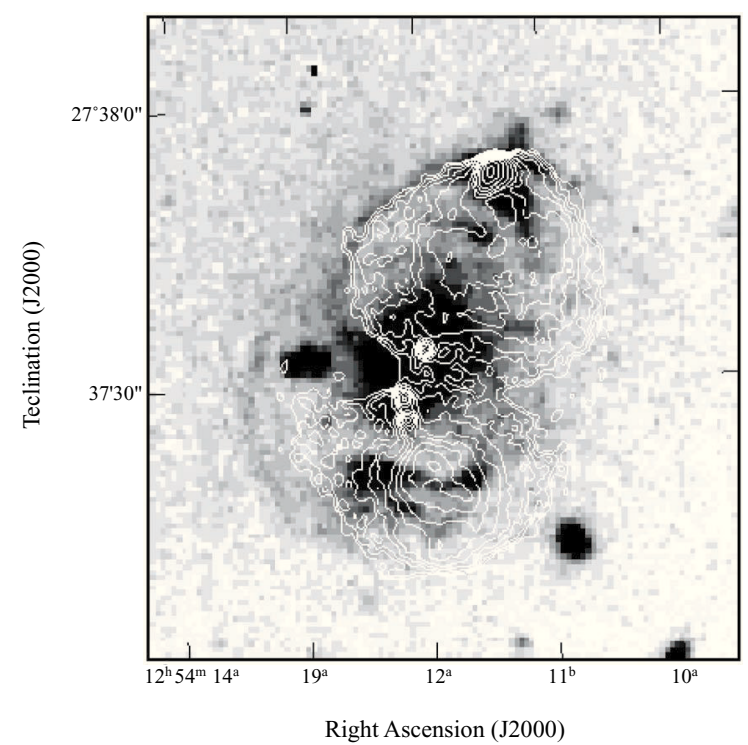

Figure 4 Deep TTF image of Coma A in $\mathrm{H} \alpha$ from Tadhunter et al. (2000). A complex spiral nebula is seen to extend over $150 \mathrm{kpc}$. The overlaid radio continuum map (contours) shows striking similarities to the distribution of ionised gas.

pronounced tidal arm structure. These authors chose two narrow bands spaced by half a bandwidth in order to study the kinematics. The entire structure appears to be in rotation about the quasar. The gas is not obviously connected with any other companions in the field. UV radiation from the quasar would have to be escaping almost isotropically to account for the ionisation. Both the source of the gas and the source of ionisation remain a mystery.

\subsection{Seyfert and Starburst Galaxies}

Seyfert galaxies often show the impact of the nuclear activity on the very large scale disk of a spatially resolved galaxy in the near field. Two of the most recent and spectacular examples are NGC 1068 and NGC 7213. Can we use the ISM as a screen to interpret what is essentially unresolved and unseen at the core?

NGC 1068 is one of the most remarkable of Seyfert galaxies in the near field. It is well known that the core harbours a concealed Seyfert 1 ('low power quasar') nucleus, but just how energetic is the source? The radio jet axis exhibits a wide range of activity (Cecil et al. 2002) but can we infer the true energetics and nuclear spectrum from multi-wavelength studies (Alexander et al. 2000; Pier \& Krolik 1993)?

Shopbell et al. (2001; see also Veilleux et al. 2001) find that the well known ionisation cone (Pogge 1988) extends up to and beyond the HI edge of the early-type spiral. The observed nebula requires rather special conditions to be detectable over such a huge extent. The most likely interpretation is that a multi-phase, vertically distended ISM is being blasted by a very energetic central source $\left(L_{\mathrm{uvx}}>5 \times 10^{43} \mathrm{erg} \mathrm{s}^{-1}\right)$. Indeed, the Chandra observations appear to show the most spectacular example to date of an 'X-ray ionisation cone' (Young et al. 2001). 


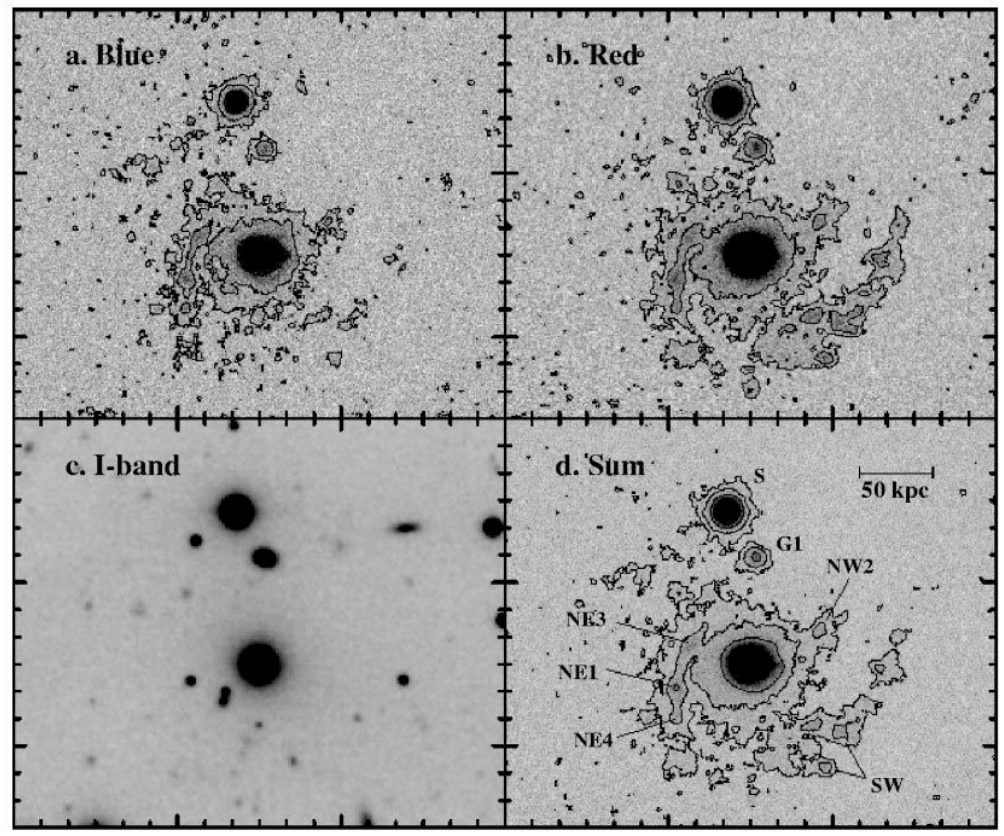

Figure 5 The extended ionised nebula surrounding the X-ray selected quasar MR 2251-178. The top figures show deep TTF H $\alpha$ images in two closely spaced narrow bands in order to emphasise the kinematic structure. The summed emission (bottom right) is seen to extend over $200 \mathrm{kpc}$ or more. The stellar continuum image is also shown (bottom left).

(a)

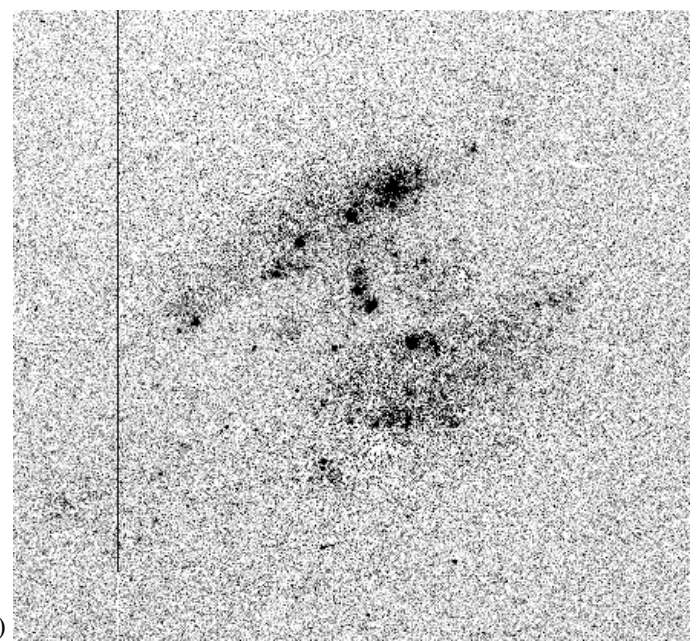

(b)

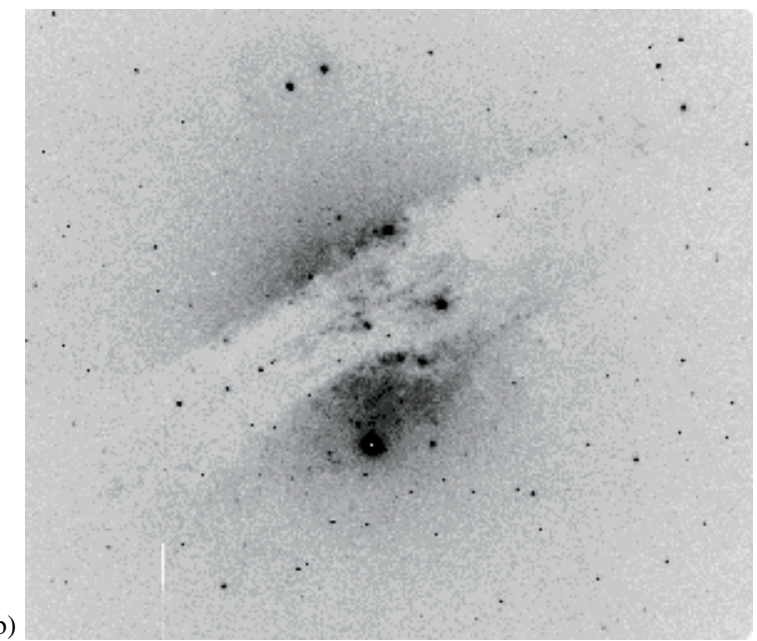

Figure 6 (a) Short exposure [OIII] $500.7 \mathrm{~nm}$ emission line halo seen above and below the dust lane in Cen A, obtained with the 'straddle shuffle' mode using the TTF (see text). These are raw data: the bad column arises from trapped charge during the charge shuffling. (b) Line + continuum image of Cen $\mathrm{A}$ in order to emphasise the dust lane.

These galaxy-scale ionisation cones are not unique to NGC 1068. Another has been detected with the TTF in NGC 7213 (Rupke et al. 2002; Veilleux et al. 2001) to rival the spectacular system in NGC 5252 studied by Tadhunter $\&$ Tsvetanov (1989). Ionisation cones are often associated with nuclear jets. Recent examples from TTF observations include IC 5063 (Cecil et al. 2001) and Circinus (Veilleux \& Bland-Hawthorn 1997). Circinus, a large spiral close to the Galactic plane, is particularly noteworthy: the system shows evidence for a whole network of 'artillery shells' blasting away from the nucleus. One such filament shows a spectacular 'Herbig Haro'-like morphology.
Extended ionisation cones are seen in a variety of sources, including the radio galaxies studied by Tadhunter (see above). One of us has obtained a short exposure [OIII] image of Cen A using the straddle shuffle mode in order to achieve a clean subtraction of the elliptical galaxy. In Figure 6, we find a very highly ionised halo of [OIII] emission above and below the dust lane. The component was first discovered by Phillips et al. (1984) who found that the faint emission appeared to rotate slowly compared to the dust lane, and typical line dispersions are hundreds of $\mathrm{km} \mathrm{s}^{-1} \mathrm{FWHM}$. We suspect that this emission arises from a highly ionised wind 
which encompasses the radio jet over a much larger solid angle.

Cecil et al. (2000) show that the famous braided jets in NGC 4258, which can be traced over $10 \mathrm{kpc}$ or more, emanate from the nuclear regions. These jets have been seen in radio continuum, Rosat/Chandra X-rays, and optical emission. They obtained deep $\mathrm{H} \alpha$ observations in the outer HI disk which gave the first detailed picture of faint tendrils of the jets. A re-analysis of the radio continuum data has revealed that these faint features have radio counterparts.

The NGC 1068 and NGC 4258 observations were able to detect faint emission unseen previously with less sensitive imaging. It is important to realise that both observations were taken within $20^{\circ}$ of the full moon, which demonstrates the effectiveness of the TTF in any phase of the lunar cycle.

A number of powerful starburst galaxies show evidence for large-scale winds along their minor axis (see reviews by Veilleux et al. 2001 and Heckman 2002). This phenomenon appears to be common at low and high redshift (Lehnert \& Heckman 1996; Veilleux et al. 2001; Pettini et al. 2001). The most detailed studies have concentrated on objects like M82, NGC 253 and NGC 3079. It remains unclear just how much energy, mass and metals these objects contribute to the intergalactic medium (IGM). In some cases, it is not clear if the wind is driven by a central starburst, an AGN or a combination of these. However there is mounting evidence that the optical diagnostics may greatly underestimate the true wind energetics (Strickland \& Stevens 2000).

Veilleux \& Rupke (2002) provide spectacular evidence for a large-scale wind in the edge on, early type galaxy NGC 1482. They obtain a clear separation of disk material from the outflowing gas. The kinematics show all the hallmarks of a biconal outflow. More impressively, the entire emission line complex has diagnostics which are entirely consistent with fast shocks. In other wind systems, there is often a large contribution from the central ionising stellar radiation field.

More recently, Veilleux et al. (2003) have presented results of a pilot imaging study of 10 nearby starburst and active galaxies conducted with the TTF on the AngloAustralian Telescope. An important goal of this study was to search for warm emission line gas on scales larger than $\sim 10 \mathrm{kpc}$ to constrain the zone of influence of these galaxies. Large-scale structures are discovered or confirmed in four of the galaxies (NGC 1068, NGC 1482, NGC 4388, NGC 6240, and NGC 7213). Unsuspected structures are also seen for the first time in the galactic winds of NGC 1365, NGC 1705, Circinus, and ESO484G036. The TTF data are combined with new optical long-slit spectra as well as published and archived radio and X-ray maps to constrain the origin and source of ionisation of these filaments. A broad range of phenomena is observed, including large-scale ionisation cones and galactic winds, tidal interaction, and ram-pressure stripping by an intracluster medium.

\subsection{Disk-Halo Connection in Spirals}

For his thesis work, Miller (2002) has undertaken an emission line survey of edge-on spiral galaxies in order to trace the connection of disk star formation with activity in galactic haloes. Miller \& Veilleux (1999) and Veilleux et al. (2001) show spectacular examples of diffuse ionised gas several kiloparsecs off the plane of normal spirals. The ratio maps show enhanced [NII] emission with respect to $\mathrm{H} \alpha$ as we move away from the plane which is most easily explained as a higher electron temperature in the halo gas (cf. Sokolowski 1993; Reynolds et al. 1999; Collins $\&$ Rand 2001). The source of the temperature increase is a topic of debate in contemporary astrophysics. Miller \& Veilleux $(2003 \mathrm{a}, \mathrm{b})$ confirm a strong correlation between the mass of ionised halo gas and the SFR per unit disk area.

\subsection{Star Formation in Elliptical and Spiral Galaxies}

Very little is known about the frequency and nature of star formation in elliptical galaxies, even though we have known for a long time that a large fraction contain cold gas (van Gorkom 1997; Knapp 1999). Ferguson and collaborators (2001) have been making $\mathrm{H} \alpha$ observations with TTF of a large sample of HI-selected ellipticals. Essentially all of these systems (see Fig. 7) show evidence of star formation to date.

For her thesis work, Cianci (2003 a,b) is undertaking a detailed comparison of 20 face-on spirals observed at $\mathrm{H} \alpha$ and $\mathrm{H} \beta$ with $\mathrm{UV}(150 \mathrm{~nm})$ images from the Ultraviolet Imaging Telescope (see Fig. 8). Her particular interest is to understand asymmetries in spiral galaxies, and the connection of the diffuse ionised gas with the HII regions and dust distribution (cf. Zurita 2001; Zurita et al. 2000).

While spiral galaxies are commonly assumed to be axisymmetric, it is surprising how few galaxies approach this ideal. The origin of asymmetries may have profound implications for their properties. An interesting possibility is that the spiral galaxy disk 'sloshes around' within the dark matter potential (e.g. Syer \& Tremaine 1996; Schoenmakers, Franx \& de Zeeuw 1997) or that the dark halo is lopsided (Jog 1999). Simulations show that the effects can survive for one or two rotation periods $\left(\sim 10^{8} \mathrm{yr}\right)$.

To date, studies of lopsidedness have concentrated on gas morphology and dynamics. But if the simulations are right, asymmetric variations should show up in the young stellar population of any lopsided spiral. Cianci et al. (2003) may now have detected an $m=1$ asymmetry in the spiral arms of the interacting galaxies M83 and M51. The UV continuum is enhanced relative to $\mathrm{H} \alpha$ along spurs that fall on the outside of the western arm. Simulations with Starburst99 indicate that these regions are older than $20 \mathrm{Myr}$, compared to the $\mathrm{H} \alpha$-bright regions which appear to be younger than $7 \mathrm{Myr}$.

NGC 2915 is a dark-matter dominated, blue compact dwarf galaxy with an HI disk extending to a radius of $15 \mathrm{kpc}$. In optical continuum it is relatively nondescript with an optical radius of only $3 \mathrm{kpc}$. No stars were known 


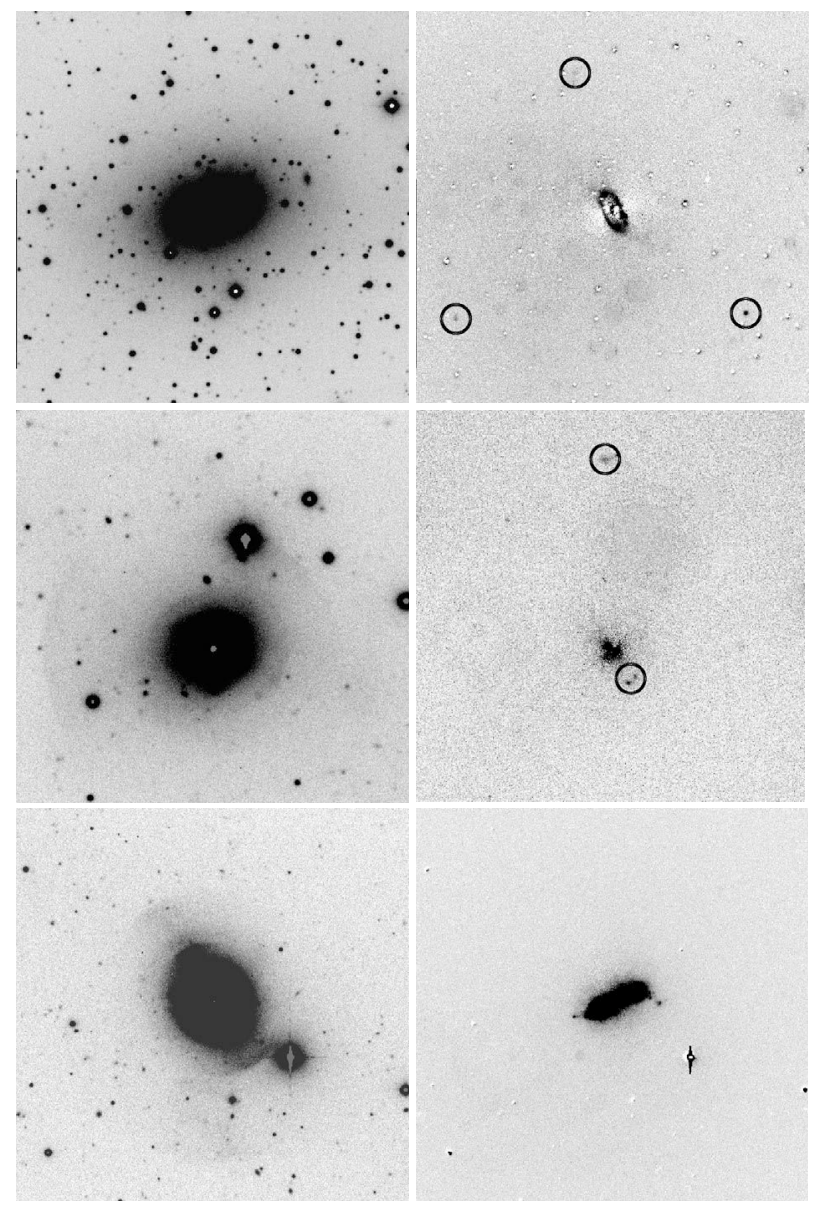

Figure $7 \quad \mathrm{R}$ band continuum images of elliptical/S0 galaxies (left) and their associated $\mathrm{H} \alpha$ emission line images (right) from the TTF survey of Ferguson et al. (2001).

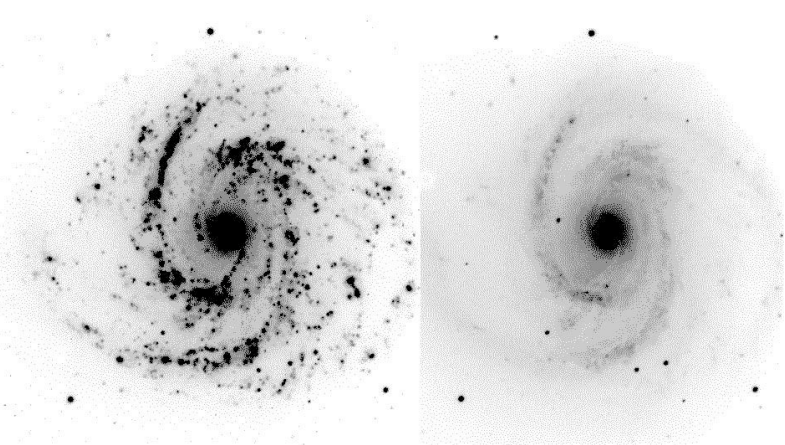

Figure 8 A demonstration of the superb contrast possible when observing a face-on spiral through a narrow band tuned to $\mathrm{H} \alpha$ (left) compared to a neighbouring continuum band (right). Both images were taken with the TTF and come from Cianci's $(2003 \mathrm{a}, \mathrm{b})$ spiral galaxy survey.

to exist beyond this radius. Meurer et al. (1999) obtained deep $\mathrm{H} \alpha$ imaging with the TTF revealing, for the first time, faint HII regions at projected radii of 3-6 kpc. These have since been confirmed with the Advanced Camera for Surveys on the Hubble Space Telescope (Meurer et al. 2003).

Higdon et al. (1997) have undertaken a multi-line study of the Cartwheel galaxy. This is the most spectacular of the class of 'ring galaxies' which are excited by the central impact of an interloper. They identify up to 100 star forming regions throughout the disk which they model as propagating star formation. There is a related class of galaxies excited by off-centre impacts. One of these, NGC 1512, has been looked at in detail with the TTF by F. Briggs (in preparation). Moreover, there is a wider class of objects under the general heading 'event driven star formation' (strong mergers, jet induced star formation, etc.) which are relatively unexplored with the TTF.

\subsection{Stellar Populations in Galaxies}

One of the most important areas where very little work has been done is imaging in stellar absorption lines. Beauchamp \& Hardy (1997) have demonstrated that this is possible even with small aperture telescopes. Molla, Hardy \& Beauchamp (1999) emphasise the importance of spatially resolved, stellar absorption line mapping of face-on spiral galaxies.

Ryder, Fenner \& Gibson (2003) have now mapped two spirals, NGC 6221 and NGC 7213, using the MgII $\lambda 5176$ and Fe $\lambda 5270$ features, two of the most prominent Lick/IDS indices (Worthey et al. 1994). The Lick spectral indices do not measure abundances per se, but can be transformed to quantities like $[\mathrm{Fe} / \mathrm{H}]$ via the method of spectral synthesis. The late-type spiral NGC 6221 shows a steep increase in both indices at the core, before dropping back to the nuclear values further out. This is in contrast to the early-type spiral NGC 7213 which has relatively constant indices in the core, followed by a smooth rise in the outer disk.

Bland-Hawthorn et al. (2001) provide a list of stellar absorption line projects which should be considered for tunable filter work.

\section{Galactic Sources}

\subsection{Pulsar Wind Nebulae}

Jones et al. (2002 a) discovered an extended $\mathrm{H} \alpha$ nebula associated with a pulsar moving rapidly through the interstellar medium. Several of these sources are now known, but in the case of pulsar B0740-28, the conic nebula is pinched perpendicular to the long axis. Possible interpretations are variations in the pulsar wind or in the external ISM.

\subsection{Interstellar Medium}

Schuberth \& Burton (2000) used the TTF to observe arguably the most exotic line to date in the optical, the [CI] $872.7 \mathrm{~nm}$ line buried deep within the $\mathrm{OH}$ forest. This line is extremely important in photodissociation regions (PDR). NGC 2023 is an HII region on the surface of a molecular cloud. These authors postulated that carbon atoms emitting at $872.7 \mathrm{~nm}$ might delineate the PDR. Their [CI] image (see Fig. 9) is the first true image of extended carbon emission ever obtained. They show that the emission correlates very closely to the so-called mysterious Extremely 

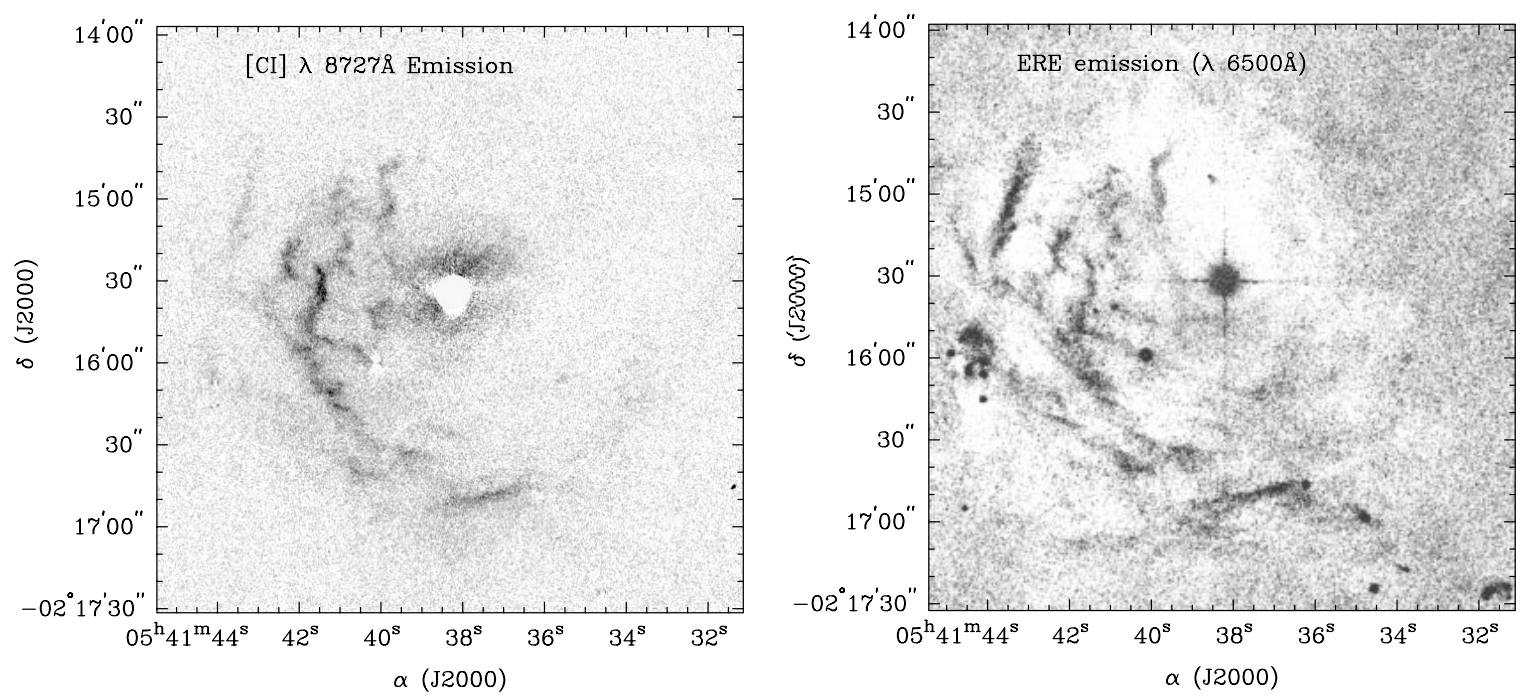

Figure 9 Schuberth \& Burton (2000) note that the left image is the first true map of carbon emission ever obtained. It was made possible by the TTF observing in an ultra-narrow band set to transmit the [CI] $872.7 \mathrm{~nm}$ emission line. Note the remarkable similarity with the Extremely Red Emission (ERE) shown on the right, although the ERE emission is generally brighter further out from the central source.

Red Emission (ERE) at about $650 \mathrm{~nm}$. [CI] is clearly a powerful tool for probing galactic star forming regions.

\subsection{Weather in Brown Dwarfs}

There is presently a lot of interest in detecting variability from brown dwarfs. If the atmospheres and environs are cool enough, models suggest that there should be dust condensations which swirl around the brown dwarf producing variability in the light curve. This was detected for the first time by Tinney \& Tolley (1999) in the M-type brown dwarf LP 944-20. They used TTF in a novel setup which required the charge to step on the CCD in synchrony with the TTF being tuned between different bands. Since then, variability has been found in another brown dwarf Kelu-1 (Clarke 2002; Clarke, Tinney \& Covey 2002). These sources do indeed appear to have complex weather patterns.

\subsection{Variable Stars}

Deutsch et al. (1998) have used TTF in time series mode to study the [OI] $844.6 \mathrm{~nm}$ emission line from the X-ray binary star V2116 Oph, the optical counterpart of GC1+4. The symbiotic-like optical spectrum of V2116 Oph shows the presence of a red giant. The X-ray source is highly variable, so the [OI] line was studied in the hope of detecting variability. This was rejected to a high level of confidence. It is thought that the system is being seen at a special time, and is probably an X-ray pulsar undergoing rapid evolution.

\subsection{Planets}

Ryder (2001) was able to image Mars in four narrow bands, a difficult experiment since the source is so bright. With four bands set at 390, 500, 668 and $707 \mathrm{~nm}$, he produced high quality images of the ice caps, the maria and terae, and various cloud formations. Jeremy Bailey
(2003, private communication) has recently emphasised the importance of tunable filters in monitoring weather patterns on Mars and Venus in narrow spectral bands. He compares the relative merits of a scanning slit, a tunable filter and an integral field spectrograph on an 8-10 m telescope. The demands on planetary science are (i) spatial stability, (ii) spatial integrity, (iii) spatial resolution of 25 mas or better, (iv) ability to switch rapidly between bands to maximise the time resolution, and to combat the planet's rotation. All of these requirements are met with a near-infrared tunable filter (resolving power $R=200$ 1000) used in conjunction with adaptive optics on a large telescope.

\section{Future Prospects}

There are numerous ways in which tunable filters can find new and important uses in astronomical programs. One possible advance for tunable filters are devices which can operate at cryogenic temperatures. Problems like piezo creep and slow gain at cryo temperatures can now be overcome.

The largest tunable filter that Queensgate can make (150 mm aperture) is not well suited to 8-10 m telescopes. But there is no reason why a $300 \mathrm{~mm}$ aperture etalon, for example, cannot be made. We have an initial design for such a system that uses an additional control stack at the centre of the plates, well matched to the central obstruction of most telescopes. This design requires a slightly reconfigured CS100 control system.

A major driver for large telescopes is diffraction limited imaging with adaptive optics. Multi-conjugate adaptive optics on a $30 \mathrm{~m}$ telescope in the J-band will permit 7 milliarcsec spatial sampling over a 2 arcmin field of view, or more than $10^{8}$ independent resolution elements. This is far beyond the capacity of an integral field spectrograph 
and can only be exploited with a tunable filter ahead of a large detector mosaic.

It is commonly thought that tunable filters are restricted to switching between bands which fall within the restricted bandpass of the order sorting filter. In fact, a multiband order sorter (Offer \& Bland-Hawthorn 1998) allows spectral bands at opposite ends of the optical spectrum to be observed in sequence (Cianci et al. 2000).

There exists a wide class of sources which have never benefitted from detailed narrowband imaging largely because even through conventional narrow bands, the field stars saturate the detector. One of many examples was an attempt to measure gas metallicities in the vicinity of the Trapezium. But rapid switching through differential ultra-narrow bands makes this entirely feasible now. The straddle shuffle demonstration by Maloney \& BlandHawthorn (2001) has been used on galaxies with very bright disks and cores, leading to perfect cancellation of the continuum light and therefore revealing very weak levels of line emission. This same method should work well on young globular clusters in order to search for $\mathrm{H} \alpha$ emission, but this has not been attempted to date.

Tunable filters have major applications for adaptive optics imaging and for coronographic imaging. The use of differential narrowband imaging would greatly help to suppress any stray light around the central or nearby bright stars. B. Woodgate has achieved some spectacular observations of emission line nebulae around symbiotic stars with the Hubble Space Telescope, but the central stars are so bright that diffraction spikes are a major problem.

There are numerous other applications, some of which require minor modifications to the existing hardware. These include new time series modes, nod \& shuffle imaging, mask \& shuffle imaging, broad-narrow switching, tunable polarimetric imaging (all discussed in BlandHawthorn 2000) and tunable echelle imaging (Baldry \& Bland-Hawthorn 2000).

\section{Appendix A: Observing Modes of Taurus}

There are web sites which describe the basic observing modes of Taurus:

Taurus homepage: www.aao.gov.au/taurus

Taurus Tunable Filter: www.aao.gov.au/ttf

Taurus++: www.aao.gov.au/taurus++

Taurus polarimetry: www.aao.gov.au/taurus_pol

\section{Appendix B: Technical Papers Relating to the TTF}

General review on tunable filters: Bland-Hawthorn (2000 a,b), Bland-Hawthorn et al. (2001)

TTF instrument summary: Bland-Hawthorn \& Jones (1998; 1999), Jones \& Bland-Hawthorn (1998), Jones (2001 a).

Charge shuffle modes: Bland-Hawthorn \& Barton (1995), Glazebrook \& Bland-Hawthorn (2001), Maloney \& Bland-Hawthorn (2001), Jones (2001 b).

TTF data analysis: Jones et al. (2002 b).

\section{References}

Alexander, T. et al. 2000, ApJ, 536, 710

Baker, J. C. et al. 2001, AJ, 121, 1821

Baldry, I. K. \& Bland-Hawthorn, J. 2000, PASP, 112, 1112

Beauchamp, D. \& Hardy, E. 1997, AJ, 113, 1666

Birkinshaw, M., Worrall, D. M. \& Hardcastle, M. J. 2001, in New Visions of the X-ray Universe, ESTEC, in press

Bland-Hawthorn, J. \& Barton, J. 1995, AAO Newsletter, 75, 8

Bland-Hawthorn, J. \& Jones, D. H. 1998, PASA, 15, 44

Bland-Hawthorn, J. \& Jones, D. H. 1998, SPIE, 3355, 855

Bland-Hawthorn, J. 2000 a, in Encyclopaedia of Astronomy \& Astrophysics, ed. P. G. Murdin IOP Publ. \& Macmillan Publ. Bristol (see http://nedwww.ipac.caltech.edu/level5/Hawthorn2/ frames.html)

Bland-Hawthorn, J. 2000 b, in Imaging the Universe in Three Dimensions, eds. van Breugel, W. \& Bland-Hawthorn, J. ASP Conf Ser, 195,34

Bland-Hawthorn, J. et al. 2001, ApJ, 563, 611

Bowyer, S., Berghöfer, T. W. \& Korpela, E. J. 1999, ApJ, 526, 592

Cecil, G. 2000, SPIE, 4008, 73

Cecil, G. et al. 2000, ApJ, 536, 675

Cecil, G. et al. 2001, BAAS, 199, 5011

Cecil, G. et al. 2002, ApJ, 568, 627

Cepa, J. et al. 2002, in Galaxies: the Third Dimension, eds. Rosada, M., Binette, L. \& Arias, L. in press

Cianci, S. 2000, AAO Newsletter, 94, 3

Cianci, S. et al. 2000, in Imaging the Universe in Three Dimensions, eds. van Breugel, W. \& Bland-Hawthorn, J. ASP Conf Ser, 195,601

Cianci, S. 2003 a, AAO Newsletter, 102, 4

Cianci, S. 2003 b, PhD, Univ. of Sydney

Cianci, S. et al. 2003, ApJ, submitted

Clarke, F. 2002, PhD, Univ. of Cambridge

Clarke, F., Tinney, C. G. \& Covey, K. R. 2002, MNRAS, 332, 361

Collins, J. \& Rand, R. 2001, ApJ, 551, 57

Couch, W. J. \& Newell, E. B. 1984, ApJS, 56, 143

Couch, W. J. et al. 2001, ApJ, 549, 820

Dalcanton, J. J. 1996, ApJ, 466, 92

Deutsch, E. W. et al. 1998, PASP, 110, 912

Dressler, A. \& Gunn, J. E. 1983, ApJ, 270, 7

Ferguson, A., van der Hulst, T. \& van Gorkom, J. 2001, AAO Newsletter, 96, 4

Francis, P., Wilson, G. M. \& Woodgate, B. E. 2001 a, PASA, 18, 64

Francis, P. et al. 2001 b, ApJ, 554, 1001

Fujita, S. S. et al. 2003, ApJ 586L,115

Glazebrook, K. 1997, in Extragalactic Astronomy in the Infrared, eds. Mamon, G. A. \& Thuan, T. X., Morimond p. 467

Glazebrook, K. \& Bland-Hawthorn, J. 2001, PASP, 113, 197

Heckman, T. 2002, in Extragalactic Gas at Low Redshift, eds. Mulchaey J. S. \& Stocke, J. T. ASP Conf Ser, 254, 292

Hewett, P. C. et al. 2000, in Imaging the Universe in Three Dimensions, eds. van Breugel, W. \& Bland-Hawthorn, J. ASP Conf Ser, 195,94

Higdon, J. L, Cecil, G. \& Bland-Hawthorn, J. 1997, AAS, 191, 8907

Hippelein, H. et al. 2003, A\&A, accepted (astro-ph/0302116)

Hu, E. M. \& McMahon, R. G. 1996, Nature, 382, 231

Jaffe, W. \& Bremer, M. 2000, AAO Newsletter, 93, 3

Jog, C. J. 1999, ApJ, 522, 661

Jones, D. H. \& Bland-Hawthorn, J. 1998, PASP, 110, 1059

Jones, D. H. \& Bland-Hawthorn, J. 1999, in Looking Deep in the Southern Sky, eds. Morganti, R. \& Couch, W. J. Berlin: SpringerVerlag p. 320

Jones, D. H. 2001, PhD, Australia National University

Jones, D. H. 2001 a, PASP, 113, 255

Jones, D. H. 2001 b, in Scientific Drivers for ESO Future VLT/VLTI Instrumentation, eds. Bergeron, J. \& G. Monnet, G.

Jones, D. H. \& Bland-Hawthorn, J. 2001, ApJ, 550, 593 
Jones, D. H., Renzini, A., Rosati, P. \& Seifert, W. 2001, ESO Mess, 103,10

Jones, D. H., Stappers, B. \& Gaensler, B. 2002 a, A\&A, 389L, 1

Jones, D. H. et al. 2002 b, MNRAS, 329, 759

Knapp, J. 1999, in Star Formation in Early-type Galaxies, eds. Carral, P. \& Cepa, J. ASP Conf. Ser., 163, 119

Lehnert, M. D. \& Heckman, T. M. 1996, ApJ, 462, 651

Lieu, R. et al. 1999, ApJ, 527, L27

Maloney, P. R. \& Bland-Hawthorn, J. 2001, ApJ, 553, L129

Meurer, G. et al. 1999, BAAS, 194, 501

Meurer, G. et al. 2003, IAU Symp. 220, Dark Matter In Galaxies, eds Walker, M \& Freeman, K.

Miller, S. T. \& Veilleux, S. 1999, AAO Newsletter, 88, 4

Miller, S. T. 2002, PhD, Univ. of Maryland

Miller, S. T. \& Veilleux, S. 2003 a, ApJS, in press (astro-ph/0305026)

Miller, S. T. \& Veilleux, S. 2003 b, ApJ, in press (astro-ph/0304471)

Miralde-Escudè, J. \& Lehar, J. 1992, MNRAS, 259, 31P

Molla, M., Hardy, E. \& Beauchamp, D. 1999, ApJ, 513, 695

Offer, A. \& Bland-Hawthorn, J. 1998, MNRAS, 299, 176

Pettini, M. et al. 2001, ApJ, 554, 981

Phillips, M. M. et al. 1984, Nature, 310, 554

Pier, E. A. \& Krolik, J. H. 1993, ApJ, 418, 673

Pogge, R. W. 1988, ApJ, 328, 519

Reynolds, R., Haffner, M. \& Tufte, S. 1999, ApJ, 525, L21

Riddick, F., Lucas, P. \& Roche, P. 2003, AAO Newsletter, 102, 10

Rupke, D. et al. 2002, in preparation

Ryder, S. 2001, AAO Newsletter, 98, 11

Ryder, S., Fenner, Y. \& Gibson, B.K. 2003, AAO Newsletter, 102, 6

Schoenmakers, A. P., Franx, M. \& de Zeeuw, P. T. 1997, MNRAS, 292, 349

Schuberth, Y. \& Burton, M. G. 2000, AAO Newsletter, 92, 3

Shopbell, P. L. et al. 1999, ApJ, 524, L83

Shopbell, P. L. et al. 2001, BAAS, 198, 5706
Smail, I. et al. 1998, MNRAS, 293, 124

Sokolowski, J. 1993, PhD, Rice University

Solorzano-Inarrea, C. et al. 2002, MNRAS, 331, 673

Solorzano-Inarrea, C. \& Tadhunter, C. N. 2003, MNRAS, 340, 705

Strickland, D. K. \& Stevens, I. R. 2000, MNRAS, 314, 511

Syer, D. \& Tremaine, S. 1996, MNRAS, 281, 925

Tadhunter, C. N. \& Tsvetanov, Z. 1989, Nature, 341, 422

Tadhunter, C. N. et al. 2000, MNRAS, 314, 849

Thommes, E. et al. 1998, MNRAS, 293, L6

Tinney, C. G. \& Tolley, A. J. 1999, MNRAS, 304, 119

Tober, J., Glazebrook, K., Thomson S., Bland-Hawthorn, J. \& Abraham, R. G. 2003, ApJ, submitted

Tresse, L., Maddox, S. J., Le Fevre, O. \& Cuby, J.-G. 2001, MNRAS, 337, 369

van Gorkom, J. 1997, in The Nature of Elliptical Galaxies, eds. Amaboldi, G. S., Da Costa, G. S. \& Saha P. ASP Conf Ser, 116, 363

Veilleux, S. \& Bland-Hawthorn, J. 1997, ApJ, 479, L105

Veilleux, S. 2002 a, in Extragalactic Gas at Low Redshift, eds. Mulchaey, J. S. \& Stocke, J. T. ASP Conf Ser, 254, 313

Veilleux, S. $2002 \mathrm{~b}$, in Galaxies: the Third Dimension, eds. Rosada, M., Binette, L. \& Arias, L. in press

Veilleux, S. \& Rupke, D. 2002, ApJ, 565, L63

Veilleux, S., Cecil, G., Bland-Hawthorn, J. \& Shopbell, P. L. 2002, in Line Emission from Jet Flows, eds. Henney, W., Steffen, W., Binette, L. \& Raga A. Rev. Mex. Astron. Astrophys., 13, 222

Veilleux, S., Shopbell, P. L., Rupke, D., Bland-Hawthorn, J. \& Cecil, G. 2003, AJ, submitted

Veilleux, S. \& Miller, S. T. 2003, AAO Newsletter, 102, 8

Young, A. J., Wilson, A. S. \& Shopbell, P. L. 2001, ApJ, 556, 6

Zaritsky, D. et al. 1997, ApJ, 480, L91

Zurita, A. 2001, PhD thesis, Instituto Astrofisica, La Laguna

Zurita, A. et al. 2000, A\&A, 363, 9 